\title{
Mental health status and perceived burden in caregiving spouses of persons with psychotic illness (A study from India)
}

\section{Selwyn Stanley PhD,} selwyn.stanley@wlv.ac.uk Senior Lecturer in Social Work, University of Wolverhampton, Wolverhampton, UK

\section{G. Mettilda Bhuvaneswari PhD} narmetm@yahoo.com Associate Professor of Social Work, Cauvery College for Women, Tiruchirappalli, India

\&

\section{S. Bhakyalakshmi MSW}

Department of Social Work, Cauvery College for Women,

Tiruchirappalli, India

Acknowledgment

The authors gratefully acknowledge permission granted by Dr. K. Ramakrishnan, Founder-Director of AIMSS (Athma Institute of Mental Health \& Social Sciences), Tiruchirappalli, India, to undertake this study and for facilitating data collection at this institution. 


\title{
Mental health status and perceived burden in caregiving spouses of persons with psychotic illness (A study from India)
}

\begin{abstract}
Mental illness poses a great deal of burden on other family members, particularly the primary caregiver. In the Indian context for a married person with mental illness, the caregiving burden is usually experienced by the spouse, who is considered to be the 'natural' option. This quantitative study used survey methodology to assess caregiving burden in fifty spouses of persons diagnosed with a psychotic illness. Data was collected at a neuro-psychiatric facility in Tiruchirappalli, India. The Depression, Anxiety and Stress Scale and the Burden Assessment Schedule were the instruments administered to assess the mental health status of the spouse and their perceived burden. Findings revealed that the majority of spouses were classified as experiencing 'severe' and 'extremely severe' in terms of their depression, anxiety and stress levels and 'high' in terms of perceived burden. The specific 'type' of clinical diagnosis, however, did not differentiate the spouses on the key variables studied. The correlation of these key variables with other background variables has also been examined. The implications of the results of this study for psychosocial intervention have also been discussed in this article.
\end{abstract}

\section{KEYWORDS}

Psychosis; caregiving burden; mental illness; spouses of persons with mental illness 
Mental illness brings with it a range of consequences not only for the individual concerned but also for the family and more specifically the primary caregiver.

Although no standard definition of caregiving exists, there is general consensus that it involves the provision of extraordinary care, exceeding the bounds of what is normative or usual in family relation-ships and typically involves significant expenditure of time, energy, and money over potentially long periods of time and involves tasks that may be unpleasant or uncomfortable and could be psychologically stressful and/or physically exhausting (Schulz \& Martire, 2004). Caregiver burden refers to the significant amount of strain and difficulties experienced by the caregiver or family member of mentally ill people and include a range of psychological, emotional, social, physical, and financial problems (Magliano, Fiorillo, De Rosa, Malangone, \& Maj, 2005). Family caregivers' burden in mental illness is a global issue and despite cultural variation is encountered by family caregivers the world over.

The World Federation of Mental Health acknowledges that caring for those with a chronic condition requires tireless effort, energy, and empathy and indisputably greatly impacts the daily lives of caregivers. Often, family caregivers receive little recognition for the valuable work that they do, and policies in most countries do not provide financial support for the care services they provide. As caregivers struggle to balance work, family, and caregiving, their own physical and emotional health is often ignored. In combination with the lack of personal, financial and emotional resources, many caregivers often experience tremendous stress, depression, and/or anxiety in the year after caregiving begins (World Federation of Mental Health, 2010).

Hoenig and Hamilton ( 1966) were the first to distinguish between sub-jective and objective burden. The former includes perceived psychological distress such as feelings of loss, embarrassment in social situations, and depression, whereas the latter includes the practical and concrete problems such as reduced social and family activities and financial difficulties.

Subjective burden scores in primary caregivers related to the behavior disturbances of the patient, social performance and adverse effects of the patient's presence on 
the household (Ricard, Bonin, \& Ezer, 1999). Family burden in caregiving of severe mental illness includes missed work, domestic routine disturbance, financial strain, effect on social and leisure activities, and decreased caring for other family members (Kreisman \& Joy, 1974). Twelve percent of caregivers of patients with a first episode of psychosis were suffering from psychiatric morbidity as assessed by the General Health Questionnaire and those living with the patient had more frequent visits to their general practitioner (Tennakoon et al., 2000). Increased family burden in psychotic patients could be associated with increased severity of the patient's mental symptoms and this impaired functioning is the single most impact on family burden (Hjarthag, Helldin, Karilampi, \& Norlander, 2010). Families with relatives diagnosed with schizophrenia, bipolar disorder, or major depression indicate that the greatest extent of burden is experienced in the following areas: worry about the future, poor concentration, upset house-hold routines, feeling guilty about not doing enough, feeling trapped, and being upset by changes in the patient (Rose, Mallinson, \& Gerson, 2006).

While the earlier literature reviewed here looked at factors contributing to caregiving burden, yet another major strand in caregiving research has been to look at the consequences of burden for the caregivers' physical and mental health. There is abundant literature that attributes caregiving strain to consequences such as depression, burn out and other forms of psychological distress (e.g. Magliano et al., 2005). Another study (Idstad, Ask, \& Tambs, 2010) shows that spouses of persons who are suffering from mental disorder report significantly higher levels of symptoms of anxiety and depression and significantly lower subjective well-being than do spouses of persons not suffering from mental disorders. Family stress is related significantly to decreased well-being, increased levels of depressive symptoms, and elevated sense of burden and that living with the care recipient is significantly associated with caregiver burden (Chen \& Lukens, 2011). Several adverse consequences are reported by family caregivers including depression, anxiety, grief, and somatic complaints, with relapses and readmissions being a com-mon feature (Chan \& Yu, 2004; Martens \& Addington, 2001).

In the Indian context, the caregiving function is usually discharged by the family, either by parents or the spouse. In marital relationships the burden of care usually 
falls on the spouse and if a woman, is perceived as a natural caregiver and one bound morally owing to religious and cultural expectations to perform this role. Often the belief in superstitions, demonic possession, and black magic drives these families to faith healers and elaborate religious rituals before the person is brought in for modern psychiatric treatment, which can be a rather expensive affair. Seeking religious help for mental disorders is often a first step in the management of mental disorders as a result of cultural explanations for the illness (Padmavati, Thara, \& Corin, 2005). Also, a visit to a psychiatric center carries a high degree of stigma and there is a general reluctance to be seen visiting a psychiatrist, counselor, or social worker. Further, the family faces a great degree of social stigma and may be shunned by neighbors and relatives. For instance the prospects of marrying into a family with a known mentally ill person are rather diminished. Marriage, fear of rejection by neighbors and the need to hide the fact from others are some of the more stigmatizing aspects (Thara \& Srinivasan, 2000). Often low literacy levels compound the ignorance about mental illness and labelling and stereotypes regarding mental illness are widely prevalent. It is against this sociocultural context that families struggle to support people who are branded as "lunatics" and "mad" in the vernacular.

Caregiving burden, especially tension, is associated with the use of maladaptive coping strategies, poor quality of life, and higher level of psychological morbidity in caregivers of patients with schizophrenia (Kate, Grover, Kulhara, \& Nehra, 2013). A comparative study from India found that caregiver burden scores in the caregivers of psychiatric patients were significantly higher than that of chronic medical illness (Ampalam, Gunturu, \& Padma, 2012). Findings from another comparative study revealed that caregivers of both long-term physical illness like intractable epilepsy and mental illness like schizophrenia experience high level of burden in areas of patient care, finance, physical and emotional burden, family relations, and occupation. However, no significant difference was found in both groups of caregivers (Sreeja, Sandhya, Rakesh, \& Singh, 2008). Caregivers of patients with bipolar disorder (BPAD) and alcohol dependence syndrome (ADS) experienced significant burden and there was no significant difference between both groups (Swapna, Sudarshan, \& Begum, 2012). The highest burden was experienced due to disruption of family activities as caregivers had to spend considerable time attending 
to the ill member at the cost of their own essential routine work/duties also resulting in the neglect of the needs of other family members (Prafulla, Murthy, \& Ramaprasad, 2010). Increase in the caregivers' burden, depression and anxiety has been shown to result in an increase in the urinary albumin: creatinine ratio indicating that psychological stress is one of the determinants of albumin excretion rate in otherwise healthy subjects (Dalui, Guha, De, Chakraborty, \& Chakraborty, 2014).

Most of the family caregiver burden literature in mental illness has revolved around people with schizophrenia, much to the exclusion of those with other chronic and severe long-term mental illnesses such as bipolar disorders or depression. The Indian literature has mostly looked at the extent of caregiving burden in mental illness such as bi-polar disorder (e.g. Chakrabarti and Gill ( 2002) and compared this with other illnesses (e.g. Chadda, Singh, and Ganguly ( 2007) or in elderly populations (e.g. Shaji, George, Prince, and Jacob ( 2009). This study was planned to understand the extent of burden experienced by the care-givers of persons diagnosed with psychosis and to ascertain their mental health status.

\section{Research questions}

The following research questions were framed:

(1) What is the extent of burden experienced by spouses of patients diagnosed with psychosis?

(2) What is the mental health status of these spouses?

(3) Is their mental health status associated with the burden owing to caregiving?

(4) What sociodemographic factors differentiate caregivers in terms of the two key subject variables (Mental health status and perceived burden)?

(5) What is the nature of association if any between sociodemographic characteristics and the key subject dimensions? 


\section{Method}

\section{Setting of the study}

The study was conducted at AIMSS (Athma Institute for Mental Health and Social Sciences; http://www.athmahospitals.com/src/main/index.php) after obtaining permission for undertaking the study. AIMSS is a psychiatric teaching cum hospital setting that offers psychiatric services to the population in and around Tiruchirappalli (Trichy) which is a central town in the south Indian state of Tamilnadu. AIMSS runs a suicide prevention clinic, has a residential rehabilitation home for mentally ill patients, offers de-addiction facilities and has university-affiliated post-graduate programs in Psychology, Psychiatry, Counselling, and Social Work. Service delivery is through a multidisciplinary team of professionals.

Respondent selection

Fifty consecutive in patients (Male-26; Female-24) who approached AIMSS were identified following diagnostic assessment by the psychiatrist according to International Classification of Diseases (ICD-10) criteria. Their spouses were asked consent to participate in the study and were included as respondents provided that they were married for at least 5 years, living with their mentally ill spouse and caring for the person at least for 1 year. The instruments were administered by the third author of the study. Data collection was undertaken between October and December 2014 and 63 patients with psychoses were admitted for inpatient services to the center of who 54 spouses met the criteria for inclusion.

Ethical clearance for the study was first obtained from the ethics review panel of AIMSS. Informed consent was then obtained from all the respondents after explaining the nature of the study to them. They were told that their participation was entirely voluntary and that they could withdraw at any point, in which case all data relating to them would be erased. They were also assured that non-participation or withdrawal would in no way affect the services that they were receiving from the 
hospital. Fifty of the carers agreed to participate in the study and were then enlisted as respondents for the study.

\section{Instruments of data collection}

A sociodemographic information sheet was prepared by the authors to collect background information and particulars relating to the illness.

The Depression, Anxiety and Stress Scale (DASS-21) is a set of three self-report scales developed by Lovibond and Lovibond ( 1995) to measure the emotional states of depression, anxiety and stress. Each of the three DASS-21 scales contains seven items, divided into subscales with similar content. The depression scale assesses dysphoria, hopelessness, devaluation of life, self-deprecation, lack of interest/involvement, anhedonia, and inertia. The anxiety scale assesses autonomic arousal, skeletal muscle effects, situational anxiety, and subjective experience of anxious affect. The stress scale is sensitive to levels of chronic non-specific arousal. It assesses difficulty relaxing, nervous arousal, and being easily upset/agitated, irritable/over-reactive and impatient. Scores for depression, anxiety and stress are calculated by summing the scores for the relevant items.

The Burden Assessment Schedule (BAS), (Sell, Thara, Padmavati, \& Kumar, 1998) is a 20 -item scale that measures subjective caregiver burden in five of the following areas was also administered: (1) Impact on well-being, (2) Impact on marital relationships, (3) Appreciation for care-giving, (4) Impact on relations with others, and (5) Perceived severity of the illness.

The BAS uses a 3-point scale for each item and can obtain a maximum score of 12 in each area of burden with higher scores indicating high degree of burden.

Both instruments were translated into the vernacular, Tamil, and back translated into English to ensure translation validity. 


\section{Results}

\section{Background profile of respondents}

The study sample had an almost equal size relating to both genders (Male-52\% and Female-48\%). The majority of the respondents were Hindus (80\%), $14 \%$ Christians and the remaining $6 \%$ were Muslims. This is generally in accordance with the demographic size of these communities in Trichy.

\section{Insert Table 1 here}

The occupational profile of the person with mental illness and the respondents are portrayed in Table 1. Clinical diagnoses received from the Psychiatrist at AIMSS shows that the major diagnostic categories according to ICD-10 criteria in which the spouses of the respondents were placed were paranoid schizophrenia (38\%), chronic schizophrenia (26\%), and depression (20\%), the other categories being bipolar affective disorders and delusional disorder ( $8 \%$ each).

The majority of respondents (68\%) had either one or two children. Thirty-six percent had a previous family history of mental illness in parents or siblings while an equal percentage said they were on regular medication. Thirty percent had undergone indigenous treatment previously, which included temple-based rituals, Ayurvedha, and Siddha (indigenous treatment modalities in India). In keeping with Indian cultural norms, the majority of the respondents were in conventional arranged marriages $(98 \%)$. Eighty percent of the respondents rated their married life as being "Good."

\section{Insert Table 2 here}

\section{Depression, anxiety, and stress}

The scores were classified according to norms suggested by the authors of the DASS in terms of severity. Table 2 shows that a relative minority were classified as being "normal" in relation to their depression (12\%), anxiety and stress (24\% each) levels. Likewise those with "mild" depression (12\%) and stress (8\%) also constituted a relatively small group with none falling in this category in terms of anxiety. The vast majority of the respondents fall in the "severe" and "extremely severe" categories 
and if these two are clubbed together constitute $54 \%$ for depression, $60 \%$ for anxiety, and $50 \%$ for stress levels.

\section{Insert Table 3 here}

\section{Perception of burden}

The respondents were re-classified into low and high categories based on the median score for the overall burden experienced as well as its sub-dimensions. With regard to the various sub-dimensions of burden, Table 3 reveals that the overall burden for the majority was "high" on the cumulative burden score (54\%) as well as all its sub-dimensions namely impact on their well-being (62\%), their marital relationship (68\%), their relationship with others (62\%), lack of appreciation for their caring role $(62 \%)$ besides the perceived severity of their spouses' ill-ness (66\%).

\section{Age-based comparison}

One way Analysis of Variance (ANOVA) was computed for the subject variables after re-classifying the respondents according to their age into four groups $(<30 ; 31-$ $40 ; 41-50 ;>50$ years). No statistical significance was seen among these age groups for all dimensions of the DASS and BAS except in terms of their perceived severity of the patient's illness $(F=2.47 ; p<.05)$. A means plot was generated for this variable and it was seen that the highest mean was for those in the 20-25 age group and the lowest for those between 51 and 55 .

\section{Insert Table 4 here}

Pearsons correlation coefficients were computed for the mean scores of the key subject variables and other selected socio-demographic variables and the results are presented in Table 4. Significant negative correlations are seen between the years of schooling (educational level) of the spouse and the overall burden score as well as the impact on their marital relationship. The age of the spouse also entered into a negative relationship with their well-being scores. The duration of marriage was positively related to the impact on the marital relationship and negatively to the wellbeing of the spouse. Also, the duration of the illness related negatively with the lack of appreciation for the caring role of the spouse. 


\section{Gender-based comparison}

t-tests were done for the variables studied comparing the gender of the respondents as well as that of their mentally ill spouses on the DASS and BAS scores. No significant gender-based differences were noticed in either comparison across all sub-dimensions.

\section{Duration of marriage}

Respondents were re-classified into three groups (<10 years; 11-20 years, and 21+ years) based on the duration of their marriage. ANOVAs for the subject variables showed significance for the well-being scores of the respondents $(F=3.77 ; p<.05)$ and lack of appreciation for caregiving ( $F=3.70 ; p<.05)$. Post hoc Scheffe comparisons showed that for both the well-being scores and the lack of appreciation for caring scores, the difference was statistically significant only for those married for less than 10 years and those in the 21 + category $(p<.05)$ than for other groups classified by their duration of marriage.

\section{Duration of mental illness}

Respondents were re-grouped according to the duration of their spouses' mental illness and those with a duration of up to 10 years compared with the $11+$ category. $t$ tests revealed statistical significance only for anxiety with a higher mean score for the $<10$ years group.

\section{Comparisons based on clinical diagnosis}

ANOVAs conducted for the DASS and BAS sub-dimensions did not elicit any statistically significant $F$ values indicating no difference in mental health status or burden experienced by the respondents based on the clinical diagnosis of their spouses. 


\section{Correlational analyses}

No statistically significant correlations were obtained between the DASS scores and those of the BAS ( $p$ > .05). It is, however, important to note that although the correlations were low they were all positive, indicating that mental health status and burden experienced improve or deteriorate in the same direction.

A few statistically significant correlations were obtained between the subject dimensions studied as well as selected sociodemographic background factors relating to the respondents. The years of schooling showed significant negative correlation with the overall burden score as well as the impact on their marital relationship. The current age of the respondents correlated negatively with their wellbeing as well as the severity of their spouses' illness but positively with the impact on their relationship with others. Their duration of being married correlated negatively to their well-being scores but positively with the impact of their relationship with others. The duration of the patients' illness correlated negatively with lack of appreciation for caring.

\section{Discussion}

It is seen that caregivers of persons with severe mental illness experience poor mental health. Higher levels of caregiver stress and anxiety has also been reported by other Indian studies (e.g., Dalui, Guha, De, Chakraborty, \& Chakraborty, 2014). This is also in consonance with the western literature on this issue (e.g., Idstad et al., 2010), which shows an association between mental illness in one person and spousal symptoms of anxiety and depression.

A study conducted in Bangalore, India recently (Swaroop et al., 2013) using the BAS that was used in the present study has reported much lower mean scores than that obtained for all BAS sub-dimensions in this study and these findings are in consonance with this study

It was seen in this study that age of the caregivers differentiated them in terms of the perceived severity of illness and this was highest for young caregivers than those 
relatively older. Age also correlated negatively with their well-being. Discriminant function analysis by Thara and Srinivasan (2000) in a study from India shows that female patients and those of a younger age for both patient and caregiver were related to higher stigma and that many care givers reported feelings of depression and sorrow. A low positive correlation for caregivers' age $<30$ years and their physical and mental health has also been reported (Srivastava, 2005).

No significant gender based differences in terms of the sex of the caregivers or their spouses and their perceived burden or mental health status was seen in this study. An earlier study from India also notes that the sex of the patient did not have a significant correlation with any of the dimensions of caregiver burden (Srivastava, 2005). This is also reported by Sreeja et al. (2008) who found no significant difference in total burden score between male and female caregivers of patients with schizophrenia. This is, however, contrary to what is known in the general literature that women tend to suffer more frequently than men from anxiety and depression (e.g. Idstad et al., 2010). The issue of social support available (not addressed by this investigation) seems to be important in the perception of burden and Hsiao (2010) reports that female family caregivers perceive less social support and experienced higher degrees of caregiver burden when compared to male family caregivers.

Anxiety levels were higher in caregivers when the spouse was ill for a shorter period ( $<10$ years) than for relatively longer spells. Perhaps longer duration of illness results in greater acceptance of it and an attitude of being reconciled to the situation. Another study from India reports that caregiver burden was found to increase with the duration of illness (Ampalam et al., 2012). However Srivastava ( 2005) found that duration of illness of $\leq 6$ months was found to have no correlation with any of the sub-dimensions of caregiver burden.

The clinical diagnosis of spouses did not differentiate between the care-givers in this study. Other studies have reported mixed findings. An Indian study (Koujalgi \& Patil, 2013) found that caregivers of patients with schizophrenia experience a high level of burden than those of patients with depressive disorders. The caregivers of patients with schizophrenia had significantly higher total burden score as compared to 
caregivers of those with a bipolar disorder. Higher burden was experienced in lack of external support, disruption of caregivers' routine and their relationships with others (Vasudeva, Sekhar, \& Rao, 2013). On the other hand, a study of partners of people suffering from anxiety disorders, depression or schizophrenia did not find any support for a relationship between strength of burden and type of diagnosis or the duration of the illness (Wittmund, Wilms, Mory, \& Angermeyer, 2002).

The negative correlation obtained between the duration of marriage and well-being scores indicate that long term suffering within their marital relationships possibly owing to their spouses' mental illness could adversely impact on their own wellbeing. Age, income or education did not show any significant correlations with depression, anxiety, or stress in this study and this is in consonance with another study (Barman \& Chakravortty, 2012).

\section{Implications for intervention}

Social workers are conventionally employed in mental health settings in India and recognized as valuable personnel within multidisciplinary teams in these settings. They work not only with patients but also their families in ushering changes that can improve the quality of life of these families. This places them in a vantage position to enable families to deal with various psychosocial issues that they face in dealing with a mentally ill member.

This study has shown that caregivers experience high levels of burden across various domains and alleviation of burden needs to be an area for psychosocial intervention. The main needs of caregivers of patients with schizophrenia relate to managing the behavior of patients; managing their social-vocational problems; health issues of caregivers; education about schizophrenia; rehabilitation; and managing sexual and marital problems of patients (Jagannathan et al., 2011). These are areas that need to be focused on during intervention and would alleviate caregiver burden as well as promote better coping with issues that they face on a day to day basis.

This study along with others makes it obvious that spouses of persons with severe 
mental illness merit therapeutic intervention in their own right in terms of their own mental health needs and this is a much overlooked domain particularly in the Indian context. Severe anxiety, depression, and stress levels have been seen in the majority of caregivers in this study. It is important that adequate intervention packages are put in place for caregiving spouses of persons who have mental illness so that their distress can be alleviated.

One to one support can be provided by social workers and counselors to enable these caregivers to work through difficulties that are encountered in their day to day life. This would enable them to deal better with the depression and anxiety that they experience.

It is also seen from the literature that social support is important for mitigating caregiving burden and so self-help groups of spouses on the lines of Alcoholics Anonymous could be another step forward. Group work sessions organized for caregivers would enable them to draw support from one another and learn coping strategies besides providing a forum for problem sharing and peer learning. Ventilation of feelings in these groups could enable them to share anxieties with one another. The need for addressing expressed emotion in comprehensive psychosocial intervention plan has also been highlighted in the literature (Nirmala, Vranda \& Reddy, 2011).

Psychoeducation is important in terms of explaining the nature of the illness, its symptoms, issues relating to relapse and the importance of treatment compliance. It is an approach that teaches patients and their families about mental disorders, their treatments, personal coping techniques and resources (Chan, 2011). So small group sessions could well be beneficial in this regard. Self-care skills, relaxation techniques, time management, budgeting are all areas that could help them deal with their worries and anxieties and promote better coping abilities. Psychoeducation has been found to reduce level of burden in caregivers of patients with schizophrenia in a pre- post intervention study in India (Sunanda, Ramesh, \& Eilean, 2013) and this is a pointer that this needs to be envisaged for all caregivers of mentally ill persons within mental health settings on a routine basis. 
Devaramane, Pai, and Vella ( 2011) provide evidence of how brief inter-ventions can bring benefits to patients as well as caregivers. They conducted brief interventions comprising of three 1-hour sessions aimed at educating the primary caregiver and patient about schizophrenia; along with improving their communication, problem solving skills and expression of emotions. A significant improvement was found between baseline and the final 3-month follow-up on measures of psychopathology for the patients, as well as family functioning for both the caregivers and patients.

Caregivers could also benefit from participation in recreational groups that could provide respite from the burden of caregiving and provide a healthy diversion from daily functions relating to caregiving.

\section{Limitations}

The study is cross-sectional in nature and does not capture the dynamics of the caregiver-patient relationship as it changes over time, thus impacting both the mental health status and perceived burden of the caregiver.

It is also unclear if better symptom management through pharmacotherapy could impact on the subject variables and influence the severity of the patient's illness. The elements of caregiver resilience, social support, and coping strategies used by caregivers are related issues that could also significantly impact the subject variables studied.

The presence of a comparative group of couples not involving a person with mental illness could have added to the empirical validity of the study.

As the respondents in this study involved persons in an inpatient clinical setting, the findings may differ for those in domiciliary community intervention programs. The findings of the study may be more relevant to Indian populations and could differ elsewhere owing to sociocultural variations, thus limiting the scope for generalization. 


\section{References}

Ampalam, P., Gunturu, S., \& Padma, V. (2012). A comparative study of caregiver burden in psychiatric illness and chronic medical illness. Indian Journal of Psychiatry, 54(3), 239-243. doi: 10.4103/0019-5545.102423

Barman, N., \& Chakravortty, P. (2012). A descriptive study to assess the level of tress among family members of selected mentally ill clients attending psychiatry OPD of a tertiary care teaching hospital. Dysphrenia, 3(1), 65-73.

Chadda, R. K., Singh, T. B., \& Ganguly, K. K. (2007). Caregiver burden and coping. Social Psychiatry and Psychiatric Epidemiology, 42(11), 923-930. doi: 10.1007/s00127-007-0242-8

Chakrabarti, S., \& Gill, S. (2002). Coping and its correlates among caregivers of patients with bipolar disorder: A preliminary study. Bipolar Disorders, 4(1), 50-60. doi: 10.1034/j.1399- 5618.2002.01167.x

Chan, S. W.-C. (2011). Global perspective of burden of family caregivers for persons with schizophrenia. Archives of Psychiatric Nursing, 25(5), 339-349. doi: 10.1016/j. apnu.2011.03.008

Chan, S., \& Yu, I. W. (2004). Quality of life of clients with schizophrenia. Journal of Advanced Nursing, 45(1), 72-83. doi: 10.1046/j.1365-2648.2003.02863.x

Chen, W.-Y., \& Lukens, E. (2011). Well being, depressive symptoms, and burden among parent and sibling caregivers of persons with severe and persistent mental illness. Social Work in Mental Health, 9(6), 397-416. doi: 10.1080/15332985.2011.575712

Dalui, A., Guha, P., De, A., Chakraborty, S., \& Chakraborty, I. (2014). Assessment of stress \& related albuminuria in caregivers of severe mentally ill persons. Indian Journal of Medical Research, 139(1), 174-177.

Devaramane, V., Pai, N. B., \& Vella, S. (2011). The effect of a brief family intervention on primary carer's functioning and their schizophrenic relatives levels of psychopathology in India. Asian Journal of Psychiatry, 4(3), 183-187. doi: 10.1016/j.ajp.2011.06.004

Hjarthag, L., Helldin, L., Karilampi, U., \& Norlander, T. (2010). Illness-related components for the family burden of relatives to patients with psychotic illness. Social Psychiatry and Psychiatric Epidemiology, 45(2), 275-283. doi: 10.1007/s00127-009-0065-x

Hoenig, J., \& Hamilton, M. W. (1966). The schizophrenic patient in the community and his effect on the household. International Journal of Social Psychiatry, 12(3), 165-176. doi: $10.1177 / 002076406601200301$

Hsiao, C.-Y. (2010). Family demands, social support and caregiver burden in Taiwanese family caregivers living with mental illness: The role of family caregiver gender. Journal of Clinical Nursing, 19(23-24), 3494-3503. doi: 10.1111/jcn.2010.19.issue-23-24

Idstad, M., Ask, H., \& Tambs, K. (2010). Mental disorder and caregiver burden in spouses: The NordTrøndelag health study. BMC Public Health, 10, 516. doi: 10.1186/1471-2458-10-516 Jagannathan,

A., Thirthalli, J., Hamza, A., Hariprasad, V. R., Nagendra, H. R., \& Gangadhar, B. N. (2011). A qualitative study on the needs of caregivers of inpatients with schizo-phrenia in India. International Journal of Social Psychiatry, 57(2), 180-194. doi: 10.1177/ 0020764009347334

Kate, N., Grover, S., Kulhara, P., \& Nehra, R. (2013). Relationship of caregiver burden with coping strategies, social support, psychological morbidity, and quality of life in the caregivers of schizophrenia. Asian Journal of Psychiatry, 6(5), 380-388. doi: 10.1016/j. ajp.2013.03.014

Koujalgi, S. R., \& Patil, S. R. (2013). Family burden in patient with schizophrenia and depressive disorder: A comparative study. Indian Journal of Psychological Medicine, 35 (3), 251-255. doi: 10.4103/0253-7176.119475 
Kreisman, D. E., \& Joy, V. D. (1974). Family response to the mental illness of a relative: A review of the literature. Schizophrenia Bulletin, 1(10), 34-57. doi: 10.1093/schbul/1.10.34

Lovibond, S. H., \& Lovibond, P. F. (1995). Manual for the depression anxiety \& stress scales (2nd ed.). Sydney, Australia: Psychology Foundation.

Magliano, L., Fiorillo, A., De Rosa, C., Malangone, C., \& Maj, M. (2005). Family burden in long-term diseases: A comparative study in schizophrenia vs. physical disorders. Social Science \& Medicine, 61(2), 313-322. doi: 10.1016/j.socscimed.2004.11.064

Martens, L., \& Addington, J. (2001). The psychological well-being of family members of individuals with schizophrenia. Social Psychiatry and Psychiatric Epidemiology, 36(3), 128- 133. doi: $10.1007 / \mathrm{s} 001270050301$

Nirmala, B. P., Vranda, M. N., \& Reddy, S. (2011). Expressed emotion and caregiver burden in patients with schizophrenia. Indian Journal of Psychological Medicine, 33(2), 119-122. doi: 10.4103/0253-7176.92052

Padmavati, R., Thara, R., \& Corin, E. (2005). A qualitative study of religious practices by chronic mentally ill and their caregivers in South India. International Journal of Social Psychiatry, 51(2), 139149. doi: $10.1177 / 0020764005056761$

Prafulla, S., Murthy, S. K., \& Ramaprasad, D. (2010). Family burden and rehabilitation: Needs of beneficiaries of a rural mental health camp in a Southern state of India. International Journal of Psychosocial Rehabilitation, 15(2), 5-11.

Ricard, N., Bonin, J.-P., \& Ezer, H. (1999). Factors associated with burden in primary caregivers of mentally ill patients. International Journal of Nursing Studies, 36(1), 73-83. doi: 10.1016/S00207489(98)00060-1

Rose, L. E., Mallinson, R. K., \& Gerson, L. D. (2006). Mastery, burden, and areas of concern among family caregivers of mentally ill persons. Archives of Psychiatric Nursing, 20(1), 41- 51. doi: 10.1016/j.apnu.2005.08.009

Schulz, R., \& Martire, L. M. (2004). Family caregiving of persons with dementia: Prevalence, health effects, and support strategies. The American Journal of Geriatric Psychiatry, 12(3), 240-249. doi: $10.1097 / 00019442-200405000-00002$

Sell, H., Thara, R., Padmavati, R., \& Kumar, S. (1998). WHO regional publication. South-East Asia Series No. 27, 13-20. Geneva: World Health Organisation.

Shaji, K. S., George, R. K., Prince, M. J., \& Jacob, K. S. (2009). Behavioral symptoms and caregiver burden in dementia. Indian Journal of Psychiatry, 51(1), 45-49. doi: 10.4103/0019-5545.44905

Sreeja, I., Sandhya, G., Rakesh, I., \& Singh, M. (2008). Comparison of burden between family caregivers of patients having schizophrenia and epilepsy. The Internet Journal of Epidemiology, 6(2). Retrieved from $\mathrm{http}: / /$ ispub.com/lJE/6/2/9378

Srivastava, S. (2005). Perception of burden by caregivers of patients with schizophrenia. Indian Journal of Psychiatry, 47(3), 148-152. doi: 10.4103/0019-5545.55938

Sunanda, G. T., Ramesh, C., \& Eilean, V. L. (2013). Effectiveness of psycho-education on care givers burden among caregivers of patients with schizophrenia. International Journal of Nursing Care, 1(2), 129-130. doi: 10.5958/j.2320-8651.1.2.048

Swapna, B., Sudarshan, C. Y., \& Begum, S. (2012). Burden on caregivers in bipolar affective disorder and alcohol dependence. International Journal of Biological Medicine Research, 3 (3), 1992-1997.

Swaroop, N., Ravi, S., Goud, B. R., Archana, M., Pius, T. M., Pal, A., . . Agrawal, T. (2013). Burden among caregivers of mentally-ill patients: A rural community-based study. International Journal of 
Research and Development of Health, 1(2), 29-34.

Tennakoon, L., Fannon, D., Doku, V., O'ceallaigh, S., Soni, W., \& Santamaria, M. (2000). Experience of caregiving: Relatives of people experiencing a first episode of psychosis. The British Journal of Psychiatry, 177(6), 529-533. doi: 10.1192/bjp.177.6.529

Thara, R., \& Srinivasan, T. N. (2000). How stigmatising is schizophrenia in India? International Journal of Social Psychiatry, 46(2), 135-141. doi: 10.1177/ 002076400004600206

Vasudeva, S., Sekhar, C. K., \& Rao, P. G. (2013). Caregivers burden of patients with schizophrenia and bipolar disorder: A sectional study. Indian Journal of Psychological Medicine, 35(4), 352-357. doi: 10.4103/0253-7176.122224

Wittmund, B., Wilms, H.-U., Mory, C., \& Angermeyer, M. C. (2002). Depressive disorders in spouses of mentally ill patients. Social Psychiatry and Psychiatric Epidemiology, 37(4), 177- 182. doi: $10.1007 / \mathrm{s} 001270200012$

World Federation of Mental Health (WFMH). (2010). Caring for the caregiver: Why your mental health matters when you are caring for others. Woodbridge, VA: WFMH. 
Table 1. Occupational status of the respondents and their spouses.

\begin{tabular}{lcc}
\hline Occupation & Patient & Spouse \\
\hline Unemployed & $15(30 \%)$ & $26(52 \%)$ \\
Coolie & $7(14 \%)$ & $3(6 \%)$ \\
Farmer & $9(18 \%)$ & $4(8 \%)$ \\
Professional & $2(4 \%)$ & $5(10 \%)$ \\
Self-employed & $8(16 \%)$ & $10(20 \%)$ \\
Clerk & $5(10 \%)$ & - \\
Government employee & $4(8 \%)$ & - \\
Teacher & - & $2(4 \%)$ \\
Total & $50(100 \%)$ & $50(100 \%)$ \\
\hline
\end{tabular}

Table 2. Distribution of respondents by severity across DASS sub-dimensions.

\begin{tabular}{|c|c|c|c|c|c|}
\hline$\rightarrow$ Severity & & & & & \\
\hline DASS variable & Normal & Mild & Moderate & Severe & Extremely severe \\
\hline Depression & $6(12 \%)$ & $6(12 \%)$ & 11 (22\%) & $5(10 \%)$ & $22(44 \%)$ \\
\hline Anxiety & 12 (24\%) & 0 & $8(16 \%)$ & 5 (10\%) & 25 (50\%) \\
\hline Stress & 12 (24\%) & $4(8 \%)$ & $9(18 \%)$ & $13(26 \%)$ & $12(24 \%)$ \\
\hline
\end{tabular}

Table 3. Distribution of respondents on sub-dimensions of BAS.

\begin{tabular}{lcrrr}
\hline Sub-dimensions & Low & High & Range & Median \\
\hline Impact on well being & $19(38 \%)$ & $31(62 \%)$ & $5-12$ & 8 \\
Impact on marital relationship & $16(32 \%)$ & $34(68 \%)$ & $5-12$ & 9 \\
Relationship with others & $19(38 \%)$ & $31(62 \%)$ & $3-9$ & 7 \\
Lack of appreciation for caring & $19(38 \%)$ & $31(62 \%)$ & $6-12$ & 10 \\
Perceived severity of illness & $17(34 \%)$ & $33(66 \%)$ & $5-12$ & 11 \\
Overall burden & $23(46 \%)$ & $27(54 \%)$ & $33-57$ & 45 \\
\hline
\end{tabular}

Table 4. Pearsons correlation coefficients for subject dimensions and select sociodemographic variables.

\begin{tabular}{|c|c|c|c|c|c|c|c|}
\hline Variables & Years of & Monthly & Present & Age at & Duration of & Number of & Duration of \\
\hline$\downarrow$ BAS \& DASS & schooling & income & age & marriage & marriage & children & mental illness \\
\hline Well being & -0.160 & 0.059 & $-0.275^{*}$ & -0.158 & $-0.271^{*}$ & -0.035 & -0.136 \\
\hline $\begin{array}{l}\text { Impact on marital } \\
\text { relationship }\end{array}$ & $-0.352^{*}$ & -0.215 & -0.093 & 0.134 & 0.001 & -0.040 & -0.112 \\
\hline $\begin{array}{l}\text { Impact on } \\
\text { relationship } \\
\text { with others }\end{array}$ & 0.006 & -0.016 & $0.369^{\star *}$ & -0.049 & $0.369^{* *}$ & 0.211 & 0.284 \\
\hline $\begin{array}{l}\text { Lack of } \\
\text { appreciation for } \\
\text { caring }\end{array}$ & -0.130 & -0.140 & -0.169 & 0.206 & -0.053 & -0.110 & $-0.233^{*}$ \\
\hline Severity of illness & -0.268 & -0.140 & $-0.315^{*}$ & -0.070 & -0.234 & -0.022 & -0.230 \\
\hline Overall BAS score & $-.0295^{*}$ & -0.134 & -0.183 & -0.022 & -0.093 & -0.005 & -0.140 \\
\hline Depression & -0.186 & -0.081 & -0.092 & 0.023 & -0.077 & 0.253 & -0.177 \\
\hline Anxiety & -0.164 & -0.166 & -0.059 & -0.049 & 0.025 & 0.228 & -0.263 \\
\hline Stress & -0.041 & -0.012 & 0.004 & 0.118 & 0.040 & 0.166 & -0.142 \\
\hline
\end{tabular}

